\title{
A SEARCH FOR GALACTIC CARBON STARS
}

\author{
Hideo Maehara \\ Tokyo Astronomical Observatory, Mitaka, Tokyo 181, Japan
}

\section{Carbon star as a probe}

The carbon star is one of the best probes for the galactic study;

(1) it is intrinsically bright $\left(M_{\mathrm{bol}}=-2\right.$ to -6$)$ especially in the red and infrared wavelength regions,

(2) it has spectral features readily detectable on objective prism plates due to their strong carbon molecular bands,

(3) it is an evolved star distributed abundantly ( 1 star per square degree) along the galactic plane.

We can detect it in the Galaxy up to several kpc from the sun on objective prism plates of the Schmidt telescope.

\section{Observation}

We have been making survey observations of faint cool carbon stars using the Kiso 105-cm Schmidt telescope. Kodak IN and 103aF plates are respectively taken behind the 4-degree objective prism ( $700 \AA^{-1} \mathrm{~mm}^{-1}$ at $\mathrm{H} \alpha$ ) for the detection and for the spectral classification. $V$-band plates are utilized to obtain the position and the brightness of the stars detected.

The survey areas are distributed along the northern galactic plane. Seven fields in the Cassiopeia region $\left(l=115^{\circ}\right.$ to $\left.133^{\circ}\right)$ and eight fields in the TaurusAuriga-Gemini region $\left(l=170^{\circ}\right.$ to $\left.188^{\circ}\right)$ have been observed and processed up to now (Maehara and Soyano 1987a,b).

\section{Detection}

The detection is made on hypersensitized IN plates covering $\lambda \lambda 6900-8800$ $\AA$, where strong $\mathrm{CN}$ bands exist in carbon stars' spectra. The limiting magnitude of our detection is around I=13 mag, and about 1 mag fainter than that of the catalog compiled by Stephenson (1973).

In total, 210 and 125 carbon stars are respectively detected in the 180 squaredegree area of the Cassiopeia region, and in the 200 square-degree area toward the galactic anticenter direction (figure 1). The mean surface number density is around 1 star per square degree at $l \sim 0^{\circ}$, and half at $l \sim 5^{\circ}$.

\section{Spectral classification}

Bright carbon stars in the Cassiopeia region are classified into the $\mathrm{C}$ classification system (Yamashita 1972,1975$)$ using $103 \mathrm{aF}$ plates $(\lambda \lambda 4500-6800$ $\AA)$. Six criteria are extracted from spectral tracings of standard stars. Fifty nine 
stars are classified into the 2-dimensional $\mathrm{C}$ system with the temperature $T$ and the carbon abundance $A$ subclasses to an accuracy of \pm 1 subclass.

Carbon stars earlier than C3 cannot be well detected due to the weakness of $\mathrm{C}_{2}$ Swan bands. A majority of the stars belong to $\mathrm{C} 4$ and $\mathrm{C} 5$ stars with high carbon abundance, while there are a few $\mathrm{C} 8$ and $\mathrm{C} 9$ stars.

\section{Space distribution}

The space distribution in the Cassiopeia region is estimated on the basis of the spectral classification and the absolute magnitudes determined by Mikami (1975). It is shown that the carbon stars are distributed over the galactic plane without strong concentration onto the (Perseus) arm. The number ratio of $\mathrm{C} 4-\mathrm{C} 5, \mathrm{C} 6-$ $\mathrm{C} 7$, and $\mathrm{C} 8-\mathrm{C} 9$ stars is nearly $1: 0.4:<0.1$.

The mean space density of cool carbon stars is about 100 stars $\mathrm{kpc}^{-3}$ in the Cassiopeia region up to $3 \mathrm{kpc}$ from the sun. The density in the anticenter region is fairly less than that of the Cassiopeia region. The space distribution of cool carbon stars is different from region to region, and the ratio to M-type giant stars is likely to be correlated with the metal abundance distribution in the Galaxy.

\section{References}

Maehara, H. 1985, Publ. Astron. Soc. Japan, 37, 333.

Maehara, H., and Soyano, T. 1987a, Ann. Tokyo Astron. Obs., 2nd Ser., 21, 293. Maehara, H., and Soyano, T. 1987b, Ann. Tokyo Astron. Obs., 2nd Ser.,21, 423. Mikami, T. 1975, Publ. Astron. Soc. Japan, 27, 445.

Stephenson, C. B. 1973, Publ. Warner Swasey Obs., 1, No.4.

Yamashita, Y. 1972, Ann. Tokyo Astron. Obs., 2nd Ser., 13, 169.

Yamashita, Y. 1975, Ann. Tokyo Astron. Obs., 2nd Ser., 15, 47.
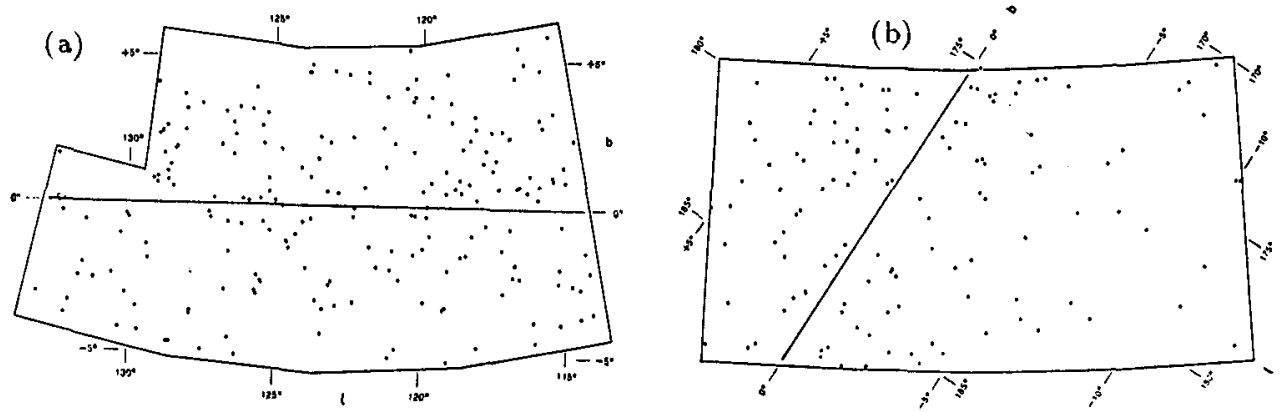

Fig. 1. Carbon stars detected in the surveyed areas.

The coordinates are given in the galactic longitude and latitude.

(a) Cassiopeia region

(b) Taurus-Auriga-Gemini region 